\title{
Assédio Moral no Trabalho: uma Revisão de Publicações Brasileiras
}

\section{Workplace Harassment: a Review of Brazilian Publications}

\author{
Júlia Gonçalves', Lucas Schweitzer², Suzana de Rosa Tolfo3
}

\begin{abstract}
Resumo
As mudanças contemporâneas ocorridas no mundo do trabalho impuseram maiores exigências aos trabalhadores e, por vezes, mostraram-se propiciadoras de diferentes violências, tais como o assédio moral no trabalho (AMT). Objetivou-se compreender como esse fenômeno vem sendo investigado por pesquisadores brasileiros. Foi realizada uma revisão de literatura de publicações indexadas nas bases de dados SciELO, PePSIC e Index-psi-periódicos. Analisou-se as características da produção científica, os procedimentos metodológicos e os objetivos e resultados dos artigos. Nos resultados destaca-se a atualidade das pesquisas e intervenções, a diversidade de objetivos e contextos investigados, bem como a necessidade de mais pesquisas que considerem a dimensão subjetiva, as características da organização, da equipe de trabalho e da sociedade para compreender o fenômeno de forma complexa. Por fim, evidencia-se a necessidade de uma visão sistêmica e de conscientizar a sociedade sobre a prevenção e o combate ao AMT.
\end{abstract}

Palavras-chave: Assédio moral. Trabalho. Violência.

\begin{abstract}
Contemporary changes in the world of work have imposed greater demands on workers and sometimes proved conducive to different forms of violence, such as moral harassment at work. This study aimed to understand how Brazilian researchers have been investigating this phenomenon. A literature review of publications indexed in Brazilian SciELO, PePSIC and Index Psi Periódicos databases was carried out. The characteristics of the production, methodological procedures, objectives and results presented in the papers were analyzed. It was possible to see the relevance of current research and interventions, the diversity of objectives and contexts investigated, as well as the need for an analysis that considers the subjective dimension, the characteristics of the organization, the work team and the society to understand the phenomenon. Finally, the data demonstrated the need for a systemic view in investigations and interventions and to raise society's awareness of the importance of preventing and tackling moral harassment.
\end{abstract}

\footnotetext{
${ }^{1}$ Universidade Federal de Santa Catarina, Santa Catarina, Brasil. E-mail: julia_psi_@hotmail.com

${ }^{2}$ Universidade Federal de Santa Catarina, Santa Catarina, Brasil. E-mail: lucass.schweitzer@gmail.com

${ }^{3}$ Universidade Federal de Santa Catarina, Santa Catarina, Brasil. E-mail: srtolfo14@gmail.com
} 


\section{Introdução}

As transformações ocorridas no contexto do trabalho trouxeram modificações nas relações de trabalho, nos modelos de gestão organizacional e na própria sociedade. Dentre as mudanças, destaca-se a intensificação da globalização, a exigência de processos de trabalho flexibilizados, o realinhamento estratégico e a modernização de processos produtivos, financeiros e administrativos (Borges \& Yamamoto, 2014; Freitas, Heloani \& Barreto, 2008) afetos ao modo de produção capitalista. Essas modificações impuseram maiores exigências aos trabalhadores, por vezes apresentadas sob a forma de fatores de risco psicossocial ou propiciadoras de diferentes violências, tais como o assédio moral no trabalho (AMT).

No fim do século XX, as violências no contexto laboral, muitas vezes naturalizadas e negligenciadas como uma construção social (Arendt, 1985; Espinosa, 2017), obtiveram mais visibilidade como fator desencadeador de sofrimento no trabalho (Freitas, Heloani, \& Barreto, 2008; Tolfo, 2011). Para Espinosa (2017), as mudanças nas formas de se relacionar e as modificações no mundo do trabalho, que geram diminuição dos postos de trabalho, favorecem os medos e alimentam o processo de banalização da violência no contexto do trabalho. No Brasil, o AMT foi reconhecido com essa denominação em 2000 (Soboll, 2015). Atualmente esse fenômeno é um importante objeto de estudo em Psicologia das Organizações e do Trabalho e o desenvolvimento de pesquisas evidencia a preocupação da comunidade científica e dos profissionais da área com o avanço do conhecimento sobre o AMT e de suas consequências sobre a saúde mental dos trabalhadores.

Por meio de um resgate histórico, identificou-se que os estudiosos escandinavos foram os primeiros a descrever o impacto e a prevalência do assédio moral (Fahie, 2014). Destaca-se, dentre eles, o psicólogo alemão Heinz Leymann, naturalizado sueco, que iniciou seus estudos sobre mobbing na década de 1980. Para ele, o fenômeno ocorre quando a vítima é submetida a estigmatização sistemática por meio de injustiças e ações frequentes e durante um longo período, ocasionando danos psíquicos, psicossomáticos e sociais. Assim, trata-se de uma comunicação hostil e antiética em direção a, principalmente, uma pessoa. Os responsáveis podem ser os colegas ou a própria gestão da organização (Leymann, 1996).

O fenômeno, como AMT, ganhou mais visibilidade quando, na mesma década, na França, passou a ser entendido e difundido por Marie France Hirigoyen como uma conduta abusiva que se manifesta, sobretudo, por comportamentos, palavras, atos e escritos que geram dano à personalidade, à dignidade e/ou à integridade física ou psíquica do alvo (Hirigoyen, 2008). Embora não se tenha pleno consenso do conceito entre os autores dedicados ao estudo do fenômeno, é possível afirmar que o AMT tem um caráter processual (Hirigoyen, 2008; Soboll, 2015), caracterizado por comportamentos repetidos e frequentes, ações e práticas abusivas expressas de diferentes formas para diminuir, humilhar, constranger, desqualificar e demolir psiquicamente um indivíduo ou grupo (Freitas, Heloani \& Barreto, 2008). 
O AMT está ligado a condutas abusivas em relação a um trabalhador, que pode gerar consequências e implicações (Hirigoyen, 2008). As atitudes hostis descritas em quatro categorias são: (i) a deterioração proposital das condições de trabalho, que inclui os atos de contestação sistemática de todas as decisões tomadas pela vítima, críticas desproporcionais, inadequadas e injustas, dentre outras; (ii) o isolamento e recusa de comunicação, tais como as atitudes de excluir a vítima; (iii) o atentado contra a dignidade, que abrange chacotas sobre características pessoais e profissionais da vítima, gestos de desprezo e injúria, dentre outras; e (iv) a violência verbal, física e sexual, como ameaças de violência física, empurrões, gritos, insinuações e assédio sexual, etc. (Hirigoyen, 2015).

No Brasil, o estudo do AMT tem início com a tradução do livro de Hirigoyen, com o título "Mal estar no trabalho: redefinindo o assédio moral", e com a dissertação de mestrado de Margarida Barreto nos anos 2000, que embora não utilizasse o termo "assédio moral", tratou da problemática sob a forma de humilhação no trabalho (Soboll, 2015). No que se refere a uma legislação nacional sobre o assunto, não se verifica maior especificidade e efetividade, com jurisdição estadual e municipais. Nogueira e Ferreira (2017) destacam algumas normatizações em relação à prática de AMT no âmbito civil, tanto na Consolidação das Leis Trabalhistas (CLT) quanto no Código Civil Brasileiro, em que é possível extrair a natureza ilícita da prática dessa violência, seja ela decorrente de um ato comissivo ou omissivo, de negligência, seja de imperícia, dado que seria ela responsável por causar danos morais ao assediado. Ainda destacam as dificuldades na formulação de normas específicas para coibir essa prática e da vítima em provar o ocorrido, ao se considerar os aspectos subjetivos atrelados ao fenômeno (Nogueira \& Ferreira, 2017).

As consequências do AMT são inúmeras e recaem, em primeira instância, sobre a vítima, em todos os domínios de sua vida, mas repercutem no nível organizacional e social. Aprofundar o conhecimento nesse tema tem relevância científica e social, como um fenômeno que precisa ser conhecido, debatido e combatido. Este trabalho objetivou analisar as publicações realizadas em três bases de dados brasileiras, SciELO.br, PePSIC e Index-psi-periódicos, por meio da plataforma BVS-PSI, e compreender como o "assédio moral no trabalho" vem sendo investigado por pesquisadores do tema no Brasil.

\section{Percurso metodológico}

Esta pesquisa caracteriza-se como uma revisão do conhecimento científico publicado em periódicos indexados no portal BVS-PSI, incluídos artigos das bases de dados: SciELO.br, PePSIC e Index-psi periódicos técnicoscientíficos. Essas bibliotecas eletrônicas permitem acessar materiais tanto do início do estudo do tema no país quanto aqueles mais atuais, além de demonstrar a produção científica de investigadores brasileiros da área na internet. Para a seleção dos trabalhos, foram incluídos apenas artigos científicos que tratassem especificamente do tema de interesse da presente revisão. Foram excluídos trabalhos decorrentes das bases bibliográficas Index Psi TESES e Index Psi LIVROS, pois a proposta do presente artigo é a análise estrita de artigos científicos.

A revisão foi operacionalizada a partir dos descritores "assédio moral no trabalho", 
"assédio psicológico no trabalho" e "violência moral no trabalho". Também foi pesquisada a expressão de busca: "assédio moral” or "assédio psicológico" or "violência moral" and "trabalho". Esses termos foram inseridos separadamente na ferramenta de busca e com o uso de aspas a fim de obter somente artigos que utilizaram a expressão exata. A busca inicial englobou os materiais publicados até o dia 31 de dezembro de 2015. Com o intuito de atualizar a revisão, em 14 de fevereiro de 2019, foi realizada nova busca incluindo artigos dos anos de 2016 e 2017

Encontrou-se inicialmente 35 textos no indexador SciELO.br, 23 no indexador PePSIC e 27 no Index-psi periódicos técnicos-científicos, num total de 85 documentos. Ao comparar as bases de dados, excluiu-se os textos repetidos e os que não estavam disponíveis na íntegra, restando 60 artigos científicos. Após a leitura e análise dos artigos selecionados, confeccionou-se um quadro sinóptico contendo: título, ano de publicação, revista, local da produção, área de conhecimento, objetivo, delineamento do estudo, características da amostra, instrumentos, análise dos dados e bibliografias utilizadas. Para compreensão dos resultados, organizou-se as informações em três categorias de análise: (i) a produção científica sobre o assédio moral no trabalho no Brasil; (ii) a escolha metodológica e a produção do conhecimento científico; e (iii) como buscam compreender o assédio moral no trabalho. Após os resultados, procede-se a discussão das informações.

\section{A produção científica sobre assédio moral no trabalho no Brasil}

Do ponto de vista cronológico, a análise dos estudos selecionados permite identificar que a primeira publicação indexada data do ano de 2001 e configura-se como um ensaio teórico que analisa o que Freitas (2001, p. 8) denomina de “duas faces do poder perverso nas organizações modernas: o assédio moral e o sexual". Ademais, os anos de 2012 e 2006 destacam-se com o maior número de produções sobre o tema: 10 e seis artigos, respectivamente. Com os descritores "assédio moral no trabalho" e "assédio moral" and "trabalho", obteve-se o maior número de produções. Essa informação indica a terminologia que vem sendo utilizada com mais frequência pelos pesquisadores para tratar do fenômeno e reflete a forma como os artigos podem ser encontrados nas bases de dados nacionais.

Ao averiguar a área de conhecimento dos autores, percebe-se prevalência de artigos com pesquisadores da área da Psicologia $(n=41)$, tanto em publicações exclusivas quanto em parceria com pesquisadores de outras áreas. Além da Psicologia, citam-se outras áreas de formação dos autores, tais como Medicina, Administração, Sociologia, Engenharia de Produção e Comunicação Social. Em 14 estudos, foram realizadas parcerias entre pesquisadores de diferentes áreas do conhecimento. Destaca-se que, mesmo com a predominância da área da Psicologia, o periódico com o maior número de artigos é a "Revista Brasileira de Saúde 
Ocupacional" ( $\mathrm{n}=11$ ), que se propõe a publicar artigos científicos no campo da Saúde e Segurança no Trabalho, o que evidencia o interesse dos pesquisadores da área da saúde ocupacional em relação ao AMT e à interdisciplinaridade do fenômeno.

Ao analisar a origem dos autores dos artigos (estado e instituição de vinculação), identificou-se que oito artigos foram produzidos em conjunto por pesquisadores de diferentes estados e instituições nacionais. Outros nove artigos foram produzidos por pesquisadores de um mesmo estado do país, porém vinculados a instituições diferentes. Já quando consideradas as pesquisas realizadas em um único estado, o local com o número mais expressivo foi São Paulo ( $n=11)$, seguido pelo estado do Rio Grande do Sul $(n=7)$ e Minas Gerais $(n=6)$. Destaca-se que a região Norte do Brasil é a que teve o menor número de artigos sobre o AMT, o que evidencia a necessidade de também desenvolver produções nessa parte do país.

Foram identificados três estudos resultantes de parcerias entre pesquisadores nacionais e internacionais (Ferreira, Mendes, Calmaro \& Blanch, 2006; Heloani, 2005; Nakamura \& Fernández, 2004) e seis publicações exclusivas de pesquisadores internacionais em revistas brasileiras, com estudos provenientes da França (Pezé, 2004), da Espanha (Justicia, Benítez Muñoz, Fernández de Haro \& Berbén, 2007; Martínez-Hernáez \& Medeiros-Ferreira, 2010; Monte, Carretero, Roldán \& Caro, 2006), de Porto Rico (Martínez-Lugo, 2006) e de uma parceria entre a Universidad de Guadalajara México - e Universitat Rovira y Virgili - Espanha (Acosta-Fernández, Torres-López, AguileraVelasco \& Parra-Osorio, 2013).
Com a realização de um levantamento das bibliografias utilizadas, é possível identificar que os 60 artigos analisados foram escritos por 161 autores, que utilizaram 2.017 referências, representando uma média de 33,61 referências por artigo completo. Dessas referências, 1.175 foram citadas no idioma de origem dos artigos, ou seja, o português, e 842 foram decorrentes de outros idiomas, principalmente o espanhol e o inglês.

Dentre os autores-referência mais citados nos artigos analisados, a autora francesa Marie-France Hirigoyen foi a protagonista, citada em 48 estudos, seguida por Heinz Leymann $(\mathrm{n}=34)$, pioneiro nos estudos sobre AMT na década de 1980 na Suécia (Ferraz \& Emmenderfer, 2015) e Stale Einarsen $(n=27)$, considerado um seguidor de Leymann (Nunes \& Lage, 2015). Dentre as referências nacionais, a autora Margarida Barreto, precursora dos estudos sobre o tema no país, é a mais citada, em 39 dos artigos analisados. Os autores José Roberto Heloani, Maria Esther Freitas e Lis Andréa Pereira Soboll são citados com frequência, estando presentes, respectivamente, em 29, 27 e 20 dos artigos.

\section{A escolha metodológica e a produção de conhecimento científico sobre AMT}

Dentre os artigos analisados, 31 são pesquisas empíricas, com destaque para estudos de caso e de levantamentos dedicados a averiguar a ocorrência do AMT em populações diversas. Há também ensaios teóricos e revisões de literatura $(\mathrm{n}=19)$, relatos de experiência $(\mathrm{n}=5)$ e estudos de adaptação e validação de instrumentos para mensuração do fenômeno (n=3). Apenas uma 
pesquisa (Garbin e Fischer, 2012) utilizou somente a análise de documentos e compreendeu os discursos sobre AMT veiculados na mídia jornalística impressa do estado de São Paulo, no período de 1990 a 2008. A proposta de Maciel e Helal (2017) diferiu das demais, pois realizaram a análise do AMT utilizando o filme "O diabo veste Prada" em sua discussão.

A maior parte dos estudos pode ser considerada de abordagem qualitativa $(n=23)$, com a utilização predominante de entrevistas como estratégia de coleta de dados $(n=12)$. Algumas pesquisas adotaram o procedimento de "histórias de vida", cujo objetivo é entender como o sujeito elabora a sua história e como se relaciona com a sociedade, em especial no trabalho (Siqueira, Saraiva, Carrieri, Lima \& Andrade, 2009). As principais formas de tratamento dos dados foram a análise de conteúdo $(n=10)$ e a análise de discurso $(n=4)$. Em 17 estudos, estavam explicitados claramente os procedimentos metodológicos adotados para a coleta de informações/dados, o que é importante para a compreensão dos dados obtidos pelos autores.

A adoção da abordagem quantitativa foi identificada em 12 estudos, sendo que três desses relacionavam-se à adaptação e validação de instrumentos. As coletas de dados foram realizadas por meio de questionários, escalas ou inventários e, como o próprio método de pesquisa sugere, foram utilizados procedimentos estatísticos para a análise dos dados.

Nos cinco relatos de experiência selecionados, as informações apresentadas foram obtidas de diversas formas, entre elas entrevistas, atendimentos (individuais e em grupo) a trabalhadores vítimas de AMT, observações e vistorias nos locais de trabalho, análises de documentos e discussões com representantes das empresas. Três dos relatos de experiência são resultados de projetos desenvolvidos por universidades para atendimento à sociedade civil (Albanaes, Rodrigues, Pellegrini \& Tolfo, 2017; Martins, Caldas, Cugnier, Goulart \& Tolfo, 2012; Vieira, Barros \& Lima, 2007), um artigo focalizou uma experiência de trabalho em uma instituição bancária (Lima, Barbosa, Mendes \& Patta, 2014), e o estudo de Salerno, Silvestre e Sabino (2011) discute as intervenções em um Centro de Referência em Saúde do Trabalhador do estado de São Paulo.

Os estudos empíricos não privilegiaram nenhuma categoria/classe profissional em específico, constatado pela diversidade de grupos de trabalhadores pesquisados, tais como vigilantes (Vieira, Lima \& Lima, 2012), trabalhadores em centrais de atendimento (Venco, 2006), professores (Rodrigues \& Freitas, 2014), profissionais de saúde (Xavier et al., 2008), agentes comunitários de saúde (Mesquita, Silva, Bezerra, Fontinele \& Neiva, 2017), trabalhadores em situação de desemprego (Scur \& Carlotto, 2012), operadores do direito (Battistelli, Amazarray \& Koller, 2011), psicólogos (Rissi, Monteiro, Cecconello \& Moraes, 2016). Apesar disso, destaca-se que três artigos foram realizados com bancários (Lima et al., 2014; Maciel, Cavalcante, Matos \& Rodrigues, 2007; Soares \& Villela, 2012), e outros três estudos com trabalhadores com Lesões por Esforços Repetitivos (Lima et al., 2014; Salerno, Silvestre \& Sabino, 2011; Silva, Oliveira \& Zambroni-deSouza, 2011). Também chama a atenção a presença de estudos que focalizam violências sofridas por mulheres (Albanaes et al., 2017; 
Antunes, Carlotto \& Strey, 2012; Valadão Júnior \& Mendonça, 2015) e homossexuais (Siqueira et al., 2009). A próxima seção dedica-se a apresentar as formas de compreender do AMT, expondo os objetivos e alguns resultados dos artigos analisados.

\section{A busca pela compreensão do fenômeno assédio moral no trabalho}

A análise dos objetivos dos artigos possibilitou identificar uma prevalência de estudos com o propósito de caracterizar, descrever, analisar ou compreender o fenômeno, tanto por meio de reflexões e ensaios teóricos (Caniato \& Lima, 2008; Freitas, 2001; Guimarães \& Rimoli, 2006; Heloani, 2005; Marangoni, Braz \& Hashimoto, 2016; Pezé, 2004) como a partir de pesquisas empíricas (Justicia et al., 2007; Maciel et al., 2007; Martínez-Lugo, 2006; Siqueira et al., 2009; Soares \& Villela, 2012; Vieira, Lima \& Lima, 2012; Xavier et al., 2008). Um exemplo é o estudo de Soares e Villela (2012) com funcionários de um banco, que identificou que o AMT, como forma de violência, é um fenômeno reconhecido pelos bancários em seu trabalho. Em geral, eles o associam à gestão por pressão, focada em metas, produção e competitividade. Outro exemplo é o estudo de Siqueira et al. (2009), que analisou as formas de violência moral no trabalho envolvendo homossexuais masculinos e identificou um contexto propício a essa violência, explícita ou velada, e de omissão dos superiores hierárquicos em relação a tais situações.

Outro destaque foram os estudos focados na caracterização do fenômeno e nas vivências das vítimas de humilhação, sofrimento, constrangimento e de AMT de forma geral, principalmente por meio de relatos, entrevistas e histórias de vida (Borsoi, Rigotto \& Maciel, 2009; Jacoby, Falcke, Lahm \& Nunes, 2009; Pinto \& Paula, 2013; Rigotto, Maciel \& Borsoi, 2010; Schlindwein, 2013; Scur \& Carlotto, 2012). O estudo de Scur e Carlotto (2012) identificou que a vivência do AMT para seis trabalhadores desempregados e tinha como pano de fundo o assédio organizacional. Já no relato das vivências de humilhação e sofrimento de trabalhadoras que participaram de um grupo terapêutico, Schlindwein (2013) identificou que a capacidade produtiva, as habilidades, a criatividade e a dedicação ao trabalho foram comprometidas a partir de situações de humilhação e constrangimento por parte da chefia e da falta de autonomia.

Ainda com o objetivo de compreender o fenômeno a partir de relato de vítimas, três estudos discorreram sobre diferentes aspectos associando o AMT com o adoecimento por Lesões por Esforços Repetitivos (LER) e Distúrbios Osteomusculares Relacionados ao Trabalho - Dort (Lima et al., 2014; Salerno, Silvestre \& Sabino, 2011; Silva, Oliveira \& Zambroni-de-Souza, 2011). Em uma das pesquisas, foi identificada a potencialização de sobrecarga mental e psíquica dos trabalhadores com LER/Dort por sujeição a pressões para que desistissem de comprovar a relação da doença com o trabalho (Lima et al., 2014).

A compreensão do AMT por meio de outros sujeitos, que não os assediados, tais como de gestores (Martiningo Filho \& Siqueira, 2008), operadores do Direito (Battistelli, Amazarray \& Koller, 2011) ou assediadores (Acosta-Fernández et al., 2013), foi objetivo de cinco estudos. O trabalho de Valadão Junior e Mendonça (2015) 
distingue-se ao analisar processos jurídicos de AMT e discursos de autores, vítimas e de gestores de organizações responsabilizadas por casos de AMT. Já a pesquisa de Garbin e Fischer (2012) analisou a temática do AMT veiculada na mídia jornalística, com destaque para discursos de natureza indenizatória, de precaução empresarial e de estratégias de enfrentamento. Indiferença, constrangimentos, desqualificações e ridicularizações foram identificadas como foco nas matérias. Outro exemplo é o estudo de Guimarães, Cançado e Lima, (2016), que caracterizou um caso de assédio moral ocorrido em uma instituição federal de ensino superior a partir da análise do processo administrativo, dos depoimentos dos envolvidos no caso, bem como por meio de uma entrevista com a vítima

O AMT também foi relacionado a outros fenômenos de pesquisa, na busca por entender relações e repercussões. Foram identificados estudos que objetivaram: analisar a associação de sintomas do mobbing com critérios de transtornos do grupo paranoide (Martínez-Hernáez \& Medeiros-Ferreira, 2010); traçar um paralelo teórico-metodológico com a retaliação no trabalho (Mendonça, Torres \& Zanini, 2008); estabelecer relações entre o AMT e a construção "identitária" (Pezé, 2004); o poder nas organizações (Freitas, 2001); a gestão e a liderança (Penteado, Mutton, Lunardelli, Goulart Júnior \& Canêo, 2011); com o bem-estar no trabalho (Jacoby \& Monteiro, 2016); e satisfação no trabalho e saúde (Silva, Aquino \& Pinto, 2017)

Quatro estudos objetivaram investigar a prevalência de AMT com diferentes públicos-alvo, sendo eles: estudantes universitários (Jacoby \& Monteiro, 2014), trabalhadores de centros de apoio a pessoas com deficiência (Monte et al.,
2006), em agentes comunitários de saúde (Mesquita et al., 2017), e trabalhadores do gasoduto Brasil-Bolívia (Guimarães, Vasconcelos, Andrade, Stephanini \& Rego, 2005). Nessa última pesquisa, o mobbing no trabalho foi correlacionado com suspeição de Transtornos Mentais, indicando-o como fator psicossocial de risco (Guimarães et al., 2005).

Um conjunto de pesquisas é dedicado ao estudo do contexto e das condições de trabalho, das ações nas organizações e das características pessoais e laborais que podem influenciar a prática do AMT (Ferreira et al., 2006; Heloani, 2005; Jacoby \& Monteiro, 2014; Rigotto, Maciel \& Borsoi, 2010; Rodrigues \& Freitas, 2014; Venco, 2006; Vieira, Lima \& Lima, 2012). O estudo de Venco (2006) destaca que a organização do trabalho, muitas vezes, estabelece condições nocivas aos trabalhadores e suscita indagações sobre os limites sutis que separam a pressão no trabalho e por produtividade e o AMT.

Alguns estudos abordam as repercussões, impactos e danos do AMT, o que demonstra a relevância de considerar suas consequências pessoais, organizacionais e sociais (Antunes, Carlotto \& Strey, 2012; Caniato \& Lima, 2008; Elgenneni \& Vercesi, 2009; Freire, 2008; Jacoby et al., 2009; Souza \& Ducatt, 2017; Vieira, Lima \& Lima, 2012). Também encontrou-se artigos que objetivam descrever as formas de atuação e combate (Martiningo Filho \& Siqueira, 2008; Salerno, Silvestre \& Sabino, 2011; Vieira, Lima \& Lima, 2012), métodos de intervenção (Glina \& Soboll, 2012), alternativas de coibição e de prevenção (Heloani, 2005; Penteado et al., 2011) ao AMT.

Dois estudos dedicaram-se à construção de instrumentos para mensuração do AMT. 
Martins e Ferraz (2011) buscaram adaptar e validar o Cuestionario de Hostigamiento Psicológico en el Trabajo de Fornés, MartínezAbascal e García de la Banda (2008) para uma amostra de trabalhadores brasileiros. $\mathrm{O}$ estudo de Rueda, Baptista e Cardoso (2015) apresenta o processo de construção da Escala Laboral de Assédio Moral (Elam). Na seção seguinte, avançase na discussão e reflexão acerca dos resultados apresentados. Já o estudo de Silva, Aquino e Pinto, (2017) avaliou as propriedades psicométricas da versão adaptada ao Brasil do Negative Acts Questionnaire - Revised (NAQ-R) originalmente construído por Einarsen e Raknes (1997).

Ainda, cabe citar três estudos que propuseram objetivos de pesquisa diferentes dos demais. O ensaio teórico de Faiman (2016) discutiu acerca da psicoterapia em situações de AMT, chamando a atenção ao manejo clínico e análise do contexto social de trabalho. $\mathrm{O}$ estudo teórico de Souza e Ducatti (2017) discute o AMT como uma forma específica de violência no trabalho, enfatizando as consequências sobre a organização da classe trabalhadora, em especial os sindicatos. Por fim, a pesquisa de Rissi et al. (2016) identifica como psicólogos, inseridos em diferentes organizações de trabalho, têm enfrentado essa violência e quais são as intervenções psicológicas que mais utilizam.

\section{Discussão}

O número expressivo de estudos teóricos e de artigos com o objetivo de caracterizar, descrever, analisar ou compreender o AMT representam a preocupação dos pesquisadores em avançar em direção a uma definição e delimitação consensual em relação ao AMT.
Percebe-se a existência de um caminho em direção ao estabelecimento de um consenso sobre a conceituação do fenômeno e uma convergência no âmbito do uso do termo "assédio moral", identificado na maior parte dos artigos analisados. Essa coerência no uso do termo contribui para a busca de uma definição precisa, importante para a atuação profissional, para o encaminhamento de casos e para o rigor científico na realização de pesquisas (Soboll, 2015).

O destaque para pesquisadores da área da Psicologia nos estudos analisados pode ser explicado pelas próprias bases de dados escolhidas para a busca, que indexam principalmente artigos da Psicologia, além de destacar uma preocupação e interesse dessa ciência no entendimento e definição do fenômeno e o fato de se tratar de uma violência predominantemente psicológica, subjetiva. O protagonismo da Psicologia coloca em questão a premissa de multidisciplinaridade teoricamente associada ao construto. Embora a "Revista Brasileira de Saúde Ocupacional", que mais publicou artigos sobre o fenômeno, tenha uma perspectiva interdisciplinar, poucos estudos analisados foram elaborados em parcerias de pesquisadores de diferentes áreas do conhecimento. Dessa forma, não se apresenta com frequência a busca dessa perspectiva inter/multidisciplinar, com a compreensão do AMT como uma faceta oriunda da violência denunciada no mundo do trabalho e com implicações para os indivíduos e influências nas suas vidas pessoal, familiar, organizacional e social (Freitas, Heloani \& Barreto, 2008). A quantidade de estudos conduzidos por pesquisadores da Psicologia também reflete a busca por um olhar mais subjetivo em relação ao 
fenômeno, com privilégio de métodos mais qualitativos, dedicados essencialmente à interpretação dos sentidos e significações referentes ao AMT (Minayo, 2012).

Salienta-se que a centralidade em aspectos de ordem psicológica ou mais subjetiva do fenômeno pode dificultar uma compreensão mais global acerca do AMT, dado que suas facetas ou variáveis podem ser analisadas por diversas perspectivas do conhecimento, na prática da pesquisa e da intervenção. Essa discussão corrobora com Freitas, Heloani e Barreto (2008) e Vieira, Lima e Lima (2012), que manifestam o receio de que o debate diante do fenômeno seja confinado a um mero conflito entre indivíduos "psicologizados" e que seja desconsiderada a responsabilidade que a organização do trabalho, as mudanças nas condições de trabalho e a dificuldade de manutenção do emprego sejam negligenciadas.

A produção de artigos nacionais que contaram com autores de diferentes estados e/ou instituições evidencia a existência de uma vinculação entre diferentes especialistas na temática, cujas parcerias nas publicações não estabelecem barreiras geográficas e/ou institucionais. Além disso, o predomínio de autores-referência internacionais nos estudos brasileiros, com destaque para a autora francesa Hirigoyen, vai ao encontro dos dados encontrados por Nunes e Lage (2015) em estudos na área de Administração, demonstrando certa coerência na produção científica dessas duas áreas do conhecimento no que tange aos principais autores citados.

Ao analisar a metodologia utilizada nas pesquisas, destaca-se o privilégio da abordagem qualitativa e do uso de entrevistas. Esse aspecto pode ser justificado quando consideramos o AMT um tópico de pesquisa sensível, ou seja, que pode mobilizar diversos conteúdos e sentimentos de risco e/ou ameaça aos envolvidos, pesquisador e pesquisado, durante as etapas de realização da pesquisa. Nesse contexto, as entrevistas qualitativas são regularmente usadas para fornecer uma riqueza de detalhes que facilita o fornecimento de uma voz para as vítimas de AMT (Fahie, 2014). Esse resultado, por outro lado, pode indicar a necessidade de maior exploração e aprofundamento metodológico nas investigações sobre o fenômeno. A ausência de pesquisas com a utilização de métodos mistos indica uma lacuna dos estudos analisados sobre o AMT que contemple tanto dados quantitativos quanto qualitativos evidencia caminhos para o avanço da produção científica sobre o assunto. Além disso, mostrou-se incipiente o número de pesquisas com a finalidade de construção e validação de instrumentos relacionados ao AMT em âmbito nacional, ferramentas importantes para a detecção e diagnóstico do fenômeno em um recorte epidemiológico.

Nas estratégias escolhidas pelos autores para execução de suas pesquisas, principalmente no que diz respeito aos procedimentos e instrumentos de coleta, apenas em um estudo (Valadão, Junior \& Mendonça, 2015), os autores utilizaram-se da triangulação de dados (Minayo, 2012). Esse procedimento possibilita um maior conhecimento do fenômeno, pois configura-se como um meio de obtenção de informações por diferentes fontes.

Quanto aos participantes das pesquisas, não é possível afirmar a prevalência de públicosalvo específicos, o que reforça a perspectiva de que o assédio se constitui em uma ampla fonte de 
pesquisa em diversos contextos (Ferraz \& Emmenderfer, 2015). Alguns estudos, no entanto, focalizam as violências sofridas por grupos que se mostram propensos a sofrerem AMT por suas características, relacionadas ao gênero dos sujeitos, por exemplo (Hirigoyen, 2008; 2015). Tal informação vai ao encontro do que afirmam Freitas, Heloani e Barreto (2008): “minorias”, tais como homossexuais e pessoas com deficiência, acabam sendo alvos por conta da indiferença e preconceito dos agressores. Além disso, em três pesquisas foi identificada a potencialização de sobrecarga mental e psíquica dos trabalhadores com LER/Dort por sujeição a pressões para que desistissem de comprovar a relação da doença com o trabalho (Lima et al., 2014). Esse resultado corrobora a ideia de que pessoas que têm algum tipo de vulnerabilidade podem se tornar vítimas mais frequentes dessas formas de violência.

Alguns estudos realizaram a análise do AMT a partir de diferentes participantes, ou seja, tanto do relato do assediado como de outras pessoas que se relacionam e são afetadas por ele. Essa informação reitera a complexidade desse fenômeno de natureza social, com elementos de ordem individual, organizacional e política. A necessidade de uma ampla análise do fenômeno, não restrita à visão da vítima ou do agressor, é reforçada pelas pesquisas dedicadas ao estudo das práticas organizacionais e das condições de trabalho que podem influenciar as práticas e as denúncias de casos de AMT. Na pesquisa de Martiningo Filho e Siqueira (2006), por exemplo, identificou-se que as empresas que têm políticas claras, canais adequados para a comunicação dos casos, estruturas de trabalho menos hierarquizadas e um clima organizacional de confiança e respeito fazem com que os profissionais se sintam mais seguros para denunciar os casos de AMT.

Essa necessidade de ampliação do escopo das pesquisas também faz referência às consequências pessoais, organizacionais e sociais do AMT. São diversos os prejuízos individuais e sociais do AMT, pois atingem a vítima em todos os domínios de sua vida, inclusive sua família e relações sociais e reverberam nas organizações, no governo e na sociedade em geral. Os efeitos que transcendem o domínio pessoal afetam negativamente a eficiência e a integridade da organização (Fahie,2014). Pode-se citar como exemplos dos prejuízos para as empresas as ausências dos trabalhadores, os custos com substituições e treinamento, a precarização do trabalho, a redução da motivação e da satisfação dos funcionários (Soares \& Villela, 2012). Essas consequências demonstram a necessidade de tratar do AMT em uma perspectiva coletiva, considerando-o uma questão de ordem social, que não se restringe ao campo individual.

\section{Considerações finais}

Com o objetivo de analisar as publicações em bases de dados brasileiras e compreender como o AMT vem sendo investigado, identificou-se que embora os comportamentos negativos de violência no trabalho possam ser tão antigos quanto o próprio trabalho, as pesquisas e intervenções sobre AMT são bastante recentes. Esse dado alerta para o fato de que o conhecimento científico carece de produções que acompanhem a complexidade dos fenômenos próprios deste momento e o crescimento do quantitativo de sujeitos assediados. 
Os estudos indicam que a discussão sobre o AMT tem repercutido em diversas regiões do país, pois não se trata de um fenômeno localizado, mas discutido por pesquisadores de diferentes instituições nacionais. Apesar disso, destaca-se a concentração de pesquisas nos estados de São Paulo e Rio Grande do Sul, contrastando com a realidade de estados da região Norte do país, onde não foram encontradas publicações relacionadas à temática. Isso evidencia uma distribuição pouco linear da produção nacional, o que oculta parte da realidade brasileira e indica cenários carentes de pesquisa e produção científica sobre o AMT.

O predomínio de publicações no campo da Psicologia alerta para o risco de, ao se tratar de uma violência predominantemente psicológica, ocorrer certa "psicologização" do fenômeno, caso não se considerem as diferentes variáveis relacionadas ao construto. A compreensão do AMT requer análises interdisciplinares e a apreensão a partir de uma perspectiva ampla que considere desde o contexto econômico e político até o contexto organizacional e do trabalho, sem deixar de destacar, evidentemente, a singularidade dos seus protagonistas.

As pesquisas indicam a existência de AMT em diferentes contextos e ocupações, configurando-se um problema de diversos tipos de organizações e níveis hierárquicos, que não se restringem a populações específicas. A diversidade de amostras dos artigos analisados demonstra a importância de entender o AMT de maneira contextualizada e abrangente. $\mathrm{O}$ avanço da produção científica, com trabalhadores de diferentes ocupações, pode ser útil para conscientizar profissionais, gestores e a sociedade acerca da existência e das consequências do fenômeno, com a possibilidade de vislumbrar formas de prevenção e combate.

Uma das contribuições trazidas por esta revisão refere-se às diversas consequências do AMT, indicando um caminho de análise para pesquisadores do assunto no que se refere a não se ater estritamente à dimensão subjetiva do fenômeno. Isso ocorre, pois as características da organização, da equipe de trabalho e da sociedade na qual o sujeito está inserido são fundamentais para a compreensão do AMT. Sugere-se a compreensão cada vez maior da complexidade do fenômeno, visto que o AMT e suas repercussões não podem ser considerados um problema individual que encontra uma única "vítima" ou um "culpado" para sua ocorrência. Pelo contrário, evidencia-se a necessidade de compreender que esse é um problema amplo e que envolve fatores de ordem econômica, organizacional, cultural e de saúde pública.

Tem-se clareza de que não foi abarcado todo o conhecimento produzido em âmbito nacional sobre esse fenômeno, porém, as informações levantadas representam uma síntese da produção científica brasileira em Psicologia até o ano de 2017. Para futuros estudos, sugere-se a ampliação da amostra de artigos para outras bases de dados, tais como as internacionais e as provenientes de outras áreas de conhecimento, como a Administração e a Medicina, não contempladas neste estudo. As informações obtidas buscaram indicar caminhos para o aprimoramento dos estudos já realizados e para novas revisões sobre o AMT. Isso se faz necessário ao considerar que a produção constante de pesquisas pode ser útil como forma de conscientização da comunidade científica, de profissionais e da população em geral, tanto no 
reconhecimento da existência e das características desse fenômeno como na construção de meios efetivos de combate a essa forma de violência.

\section{Referências}

Acosta-Fernández, M., Torres-López, T. M., Aguilera-Velasco, M. de L. Á., \& ParraOsorio, L. (2013). El Acoso psicológico laboral desde la mirada de un perpetrador: un estudio de caso. Revista Mal-estar $e$ Subjetividade, 13(1-2), 13-43.

Albanaes, P., Rodrigues, K. J., Pellegrini, P. G., \& Tolfo, S. da R. (2017). Intervenção em grupo de apoio psicológico a trabalhadores vítimas de assédio moral. Revista de Psicología (PUCP), 35(1). Doi: 10.18800/psico.201701.003.

Antunes, B. M., Carlotto, M. S., \& Strey, M. N. (2012). Mulher e trabalho: visibilizando o tecido e a trama que engendram o assédio moral. Psicologia em Revista, 18(3), 420-445. Doi: 10.5752/P.16789563.2012v18n3p420.

Arendt, H. (1985). Da violência. Brasilia: Editora Universidade de Brasília.

Battistelli, B. M., Amazarray, M. R., \& Koller, S. H. (2011). O assédio moral no trabalho na visão de operadores do direito. Psicologia \& Sociedade, 23(1), 35-45. Doi: 10.1590/S0102-71822011000100005.

Borges, L. O., \& Yamamoto, O. H. (2014). Mundo do trabalho: construção histórica e desafios contemporâneos. In J. C. Zanelli, J. Borges-Andrade \& A. V. B. Bastos. Psicologia, organizacões e trabalho no Brasil (2a ed., pp. 25-72). Porto Alegre: ArtMed.
Borsoi, I. C. F., Rigotto, R. M., \& Maciel, R. H. (2009). Da excelência ao lixo: humilhação, assédio moral e sofrimento de trabalhadores em fábricas de calçados no Ceará. Cadernos de Psicologia Social do Trabalho, 12(2), 173-187. Doi: 10.11606/issn.19810490.v12i2p173-187.

Câmara, R. de A., Maciel, R. H., \& Gonçalves, R. C. (2012). Prevenção e combate ao assédio moral entre servidores públicos do estado do Ceará. Revista Brasileira de Saúde Ocupacional, 37(126), 243-255. Doi: 10.1590/S0303-76572012000200006.

Caniato, A. M. P., \& Lima, E. da C. (2008). Assédio moral nas organizações de trabalho: perversão e sofrimento. Cadernos de Psicologia Social do Trabalho, 11(2), 177192. Doi: $10.11606 /$ issn.19810490.v11i2p177-192.

Einarsen, S., \& Raknes, B. I. (1997). Harassment in the workplace and the victimization of men. Violence and Victim, 12, 247-263.

Elgenneni, S. M. M., \& Vercesi, C.(2009). Assédio moral no trabalho: implicações individuais, organizacionais e sociais. Revista Psicologia Organizações e Trabalho, 9(1), 68-85.

Espinosa, L. M. C. (2017, Maio). Prácticas en el trabajo y violencia. Conferência, Barcelona.

Fahie, D. (2014). Doing sensitive research sensitively: ethical and methodological issues in researching workplace bullying. International Journal of Qualitative Methods, 13, 19-36.

Doi: $10.1177 / 160940691401300108$.

Faiman, C. J. S. (2016). A queixa de assédio moral no trabalho e a psicoterapia. Psicologia em Estudo, 21(1), 127-135. Doi: 10.4025/psicolestud.v21i1.28311. 
Ferraz, N. D., \& Emmendoerfer, M. L. (2015). Produção internacional sobre assédio moral em organizações públicas e sindicatos. In M. L. Emmendoerfer, S. R. Tolfo \& T. S. Nunes (Orgs.). Assédio moral em organizações públicas e a (re)ação dos sindicatos (pp. 67-78). Curitiba, PR: CRV.

Ferreira, J. B., Mendes, A. M., Calgaro, J. C. C., \& Blanch, J. M. (2006). Situações de assédio moral a trabalhadores anistiados políticos de uma empresa pública. Psicologia em Revista, 12(20), 215-233.

Fornés, J., Martínez-Abascal, M. A., \& García de la Banda, G. (2008). Análisis factorial del cuestionario de hostigamiento psicológico en el trabajo en profesionales de enfermaria. Internacional Journal of Clinical and Health Psychology, 8(1), 267-283.

Freire, P. A. (2008). Assédio moral e saúde mental do trabalhador. Trabalho, educação e saúde, 6(2), 367-380. Doi: 10.1590/S198177462008000200009 .

Freitas, M. E., Heloani, R., \& Barreto, M. (2008). Assédio moral no trabalbo. São Paulo: Cengage Learning.

Garbin, A. de C., \& Fischer, F. M. (2012). Assédio moral no trabalho e suas representações na mídia jornalística. Revista Saúde Pública, 46(3), 417-424. Doi: 10.1590/S003489102012005000035.

Glina, D. M. R., \& Soboll, L. A. (2012). Intervenções em assédio moral no trabalho: uma revisão da literatura. Revista brasileira de saúde ocupacional, 37(126), 269283. Doi: $10.1590 /$ S030376572012000200008.

Guimarães, C. A., Cançado, V. L., \& Lima, R. de J. C. (2016). Workplace moral harassment and its consequences: a case study in a federal higher education institution. Revista de Administração (São Paulo), 51(2), 151-164. Doi: $10.5700 /$ rausp1231.

Guimarães, L., Vasconcelos, É., Andrade, P., Stephanini, I., \& Rego, R. (2005). Mobbing (assédio psicológico): relações com transtornos mentais e coping em trabalhadores do gasoduto Brasil-Bolívia. Revista Psicologia: Organizações e Trabalho, 5(2), 15-34.

Guimarães, L. A. M., \& Rimoli, A. O. (2006). "Mobbing" (assédio psicológico) no trabalho: uma síndrome psicossocial multidimensional. Psicologia: Teoria e Pesquisa, 22(2), 183-191. Doi: 10.1590/S010237722006000200008 .

Heloani, R. (2005). Assédio moral: a dignidade violada. Aletheia, (22), 101-108.

Hirigoyen, M. F. (2008). Assédio moral: a violência perversa no cotidiano (10a ed.). Rio de Janeiro: Bertrand Brasil.

Hirigoyen, M. F. (2015). Mal estar no trabalho: redefinindo o assédio moral (8a ed.). Rio de Janeiro: Bertrand Brasil.

Jacoby, A. R., \& Monteiro, J. K. (2016). Assédio moral em estudantes trabalhadores e sua relação com o bem-estar no trabalho. Revista Interação Psicol., Curitiba, 20(3), 319329. Doi: 10.5380/psi.v20i3.29684.

Jacoby, A. R., \& Monteiro, J. K. (2014). Mobbing of working students. Paidéia (Ribeirão Preto), 24(57), 39-47. Doi: 10.1590/198243272457201406.

Jacoby, A. R., Falcke, D., Lahm, C. R., \& Nunes, G. J. (2009). Assédio moral: uma guerra invisível no contexto empresarial. Revista Mal Estar e Subjetividade, 9(2), 619-645. 
Justicia, F. J., Benítez Muñoz, J. L., Fernández De Haro, E., \& Berbén, A. G. (2007). El fenómeno del acoso laboral entre los trabajadores de la universidad. Psicologia em Estudo, 12(3), 457-463. Doi: 10.1590/s1413-73722007000300002.

Leymann, H. (1996). The content and development of mobbing at work. European Journal of Work and Organizational Psychology, 5(2), 165-184. Doi: $10.1080 / 13594329608414853$.

Lima, C. Q. B., Barbosa, C. M. G., Mendes, R. W. B., \& Patta, C. A. (2014). Assédio moral e violências no trabalho: caracterização em perícia judicial. Relato de experiência no setor bancário. Revista Brasileira de Saúde Ocupacional, 39(129), 101-110. Doi: 10.1590/0303-7657000050313.

Maciel, H. W. P., \& Helal, D. H. (2017). "Detalhes de sua incompetência não me interessam": $\mathrm{o}$ assédio moral no filme $\mathrm{O}$ diabo veste Prada. Read. Revista Eletrônica de Administração (Porto Alegre), 23(spe), 412427. Doi: 10.1590/1413-2311.181.75135.

Maciel, R. H., Cavalcante, R., Matos, T. G. R., \& Rodrigues, S. (2007). Auto relato de situações constrangedoras no trabalho e assédio moral nos bancários: uma fotografia. Psicologia \& Sociedade, 19(3), 117-128. doi: 10.1590/S010271822007000300016 .

Marangoni, V., Braz, M., \& Hashimoto, F. (2016). Bullying e assédio moral no trabalho: expressões do narcisismo contemporâneo. Cadernos de Psicologia Social do Trabalho, 19(2), 255-268. Doi: 1981-0490.v19i2p255-268. Martínez-Hernáez, Á., \& Medeiros-Ferreira, L. (2010). Anatomía de una confusión: error diagnóstico de patología paranoide en víctimas de mobbing. Revista de Psiquiatria Clinica, 37(4), 167-174. Doi: 10.1590/S0101-60832010000400005.

Martínez-Lugo, M. E. (2006). El acoso psicológico en el trabajo: la experiencia en Puerto Rico. Aletheia, 24, 21-33.

Martiningo Filho, A., \& Siqueira, M. V. S. (2008). Assédio moral e gestão de pessoas: uma análise do assédio moral nas organizações e o papel da área de gestão de pessoas. RAM, Revista Administração Mackenzie, 9(5), 11-34. Doi: 10.1590/s1678-69712008000500002.

Martins, F. S., Caldas, D. B., Cugnier, J. S., Goulart, L., \& Tolfo, S. da R. (2012). Restabelecendo o poder de agir: atendimento grupal para assediados moralmente no trabalho. Revista Brasileira de Psicodrama, 20(2), 97-113.

Martins, M. do C. F., \& Ferraz, A. M. S. (2011). Propriedades psicométricas das escalas de assédio moral no trabalho: percepção e impacto. Psico-USF (Impr.), 16(2), 163-173. Doi: 10.1590/s1413-82712011000200005.

Mendes, E. R. P. (2012). O mal-estar nas relações de trabalho. Estudos de Psicanálise. 38, 59-64.

Mesquita, A. A., Silva, A. S., Bezerra, H. R., Fontinele, T. P., \& Neiva, Y. P.. (2017). Assédio moral: impacto sobre a saúde mental e o envolvimento com trabalho em agentes comunitários de saúde. Revista Psicologia e Saúde, 9(1), 3-17. Doi: 10.20435/pssa.v9i1.375.

Minayo, M. C. S. (Org.). (2012). Pesquisa social: teoria, método e criatividade (31a ed.). Petrópolis, RJ: Vozes. 
Monte, P. R. G., Carretero, N., Roldán, M. D., \& Caro, M. (2006). Estudio piloto sobre la prevalencia del acoso psicológico (mobbing) en trabajadores de centros de atención a personas con discapacidad. Aletheia, 23, 7-16.

Nakamura, A. P., \& Fernández, R. A. (2004). Assédio moral. Aletheia, 19, 69-74.

Nogueira, R. M., \& Ferreira, R. R. (2017). A sistematização legislativa do assédio no âmbito das Administrações Públicas Estaduais no Brasil. EnGPR 2017, Curitiba, Brasil.

Nunes, T. S., \& Lage, M. L. C. (2015). O que tem sido escrito na área de Administração sobre assédio moral no trabalho no Brasil?. In M. L. Emmendoerfer, S. R. Tolfo \& T. S. Nunes. Assédio moral em organizações públicas e a (re)ação dos sindicatos (pp.79-96). Curitiba: CRV.

Penteado, A. C. M., Mutton, M. R., Lunardelli, M. C. F., Goulart Júnior, E., \& Canêo, L. C. (2011). Liderança e assédio moral: a administração perversa do sentido do trabalho. Psicologia para América Latina, (21), 71-82.

Pezé, M-G. (2004). Forclusão do feminino na organização do trabalho: um assédio de gênero. Produção, 14(3), 6-13. Doi: 10.1590/s0103-65132004000300002.

Pinto, R. de A. B., \& Paula, A. P. P. de (2013). Do assédio moral à violência interpessoal: relatos sobre uma empresa júnior. Cadernos EBAPE.BR, 11(3), 340$355 . \quad$ Doi: 10.1590/s167939512013000300002.

Rigotto, R. M., Maciel, R. H., \& Borsoi, I. C. F. (2010). Produtividade, pressão e humilhação no trabalho: os trabalhadores e as novas fábricas de calçados no Ceará. Revista Brasileira de Saúde Ocupacional, 35(122), 217-228. Doi: 10.1590/s030376572010000200005.

Rissi, V., Monteiro, J. K., Cecconello, W. W., \& Moraes, E. G. de. (2016). Intervenções psicológicas diante do assédio moral no trabalho. Temas em Psicologia, 24(1), 339-352. Doi: 10.9788/TP2016.1-2.

Rodrigues, M., \& Freitas, M. E. de. (2014). Assédio moral nas instituições de ensino superior: um estudo sobre as condições organizacionais que favorecem sua ocorrência. Cadernos EBAPE.BR, 12(2), 284-301. Doi: 10.1590/1679-39518275.

Salerno, V. L., Silvestre, M. P., \& Sabino, M. O. (2011). Interfaces LER/saúde mental: a experiência de um Centro de Referência em Saúde do Trabalhador do Estado de São Paulo. Revista Brasileira de Saúde Ocupacional, 36(123), 128-138. Doi: 10.1590/s0303-76572011000100012.

Schlindwein, V. de L. D. C. (2013). Histórias de vida marcadas por humilhação, assédio moral e adoecimento no trabalho. Psicologia \& Sociedade, 25(2), 430-439. Doi: 10.1590/s0102-71822013000200020.

Scur, M. D., \& Carlotto, M. S. (2012). Assédio moral: a percepção e vivência dos trabalhadores. Contextos Clínicos, 5(2), 121132. Doi: $10.4013 /$ ctc.2012.52.06.

Silva, E. F. da, Oliveira, K. K. M. de, \& Zambronide-Souza, P. C. (2011). Saúde mental do trabalhador: o assédio moral praticado contra trabalhadores com LER/Dort. Revista Brasileira de Saúde 
Ocupacional, 36(123), 56-70. Doi: 10.1590/s0303-76572011000100006.

Silva, I. V., Aquino, E. M. L. de, \& Pinto, I. C. de M. (2017). Características psicométricas do Negative Acts Questionnaire para detecção do assédio moral no trabalho: estudo avaliativo do instrumento com uma amostra de servidores estaduais da saúde. Revista Brasileira de Saúde Ocupacional, 42, e2. Epub March 13.

Siqueira, M. V. S., Saraiva, L. A. S., Carrieri, A. de P., Lima, H. K. B. de, \& Andrade, A. J. de A. (2009). Homofobia e violência moral no trabalho no Distrito Federal. Organizações \& Sociedade, 16(50), 447-461. Doi: 10.1590/s1984-92302009000300003.

Soares, L. R., \& Villela, W. V. (2012). O assédio moral na perspectiva de bancários. Revista Brasileira de Saúde Ocupacional, 37(126), 203212. Doi: 10.1590/s030376572012000200003.

Soares, L. Q., \& Ferreira, M. (2006). Pesquisa participante como opção metodológica para investigação de práticas de assédio moral no trabalho. Revista Psicologia Organizações e Trabalho, 6(2), 85-109.

Soboll, L. A. P. (2015). Assédio moral no trabalho. In P. F. Bendassolli \& J. E. Borges-Andrade. Dicionário de Psicologia do Trabalho e das Organiz̧ações (pp. 85-93). São Paulo: Casa do psicólogo.

Sousa, A. M. (2014). A consagração das vítimas nas sociedades de segurança. Revista EPOS, 5(1), 29-56.

Souza, T. M. dos S., \& Ducatti, I.. (2017). O enfrentamento do assédio moral pelos sindicatos: contribuições marxistas. Cadernos de Psicologia Social do Trabalho, 20(1),
79-94. Doi: 10.11606/issn.19810490.v20i1p79-94.

Tolfo, S. da R. (2011). O assédio moral como expressão da violência no trabalho. In M. Souza, F., Martins \& J. N. G. Araújo (Orgs.). Dimensões da violência: conhecimento, subjetividade e sofrimento psíquico (pp.187-206). São Paulo: Casa do Psicólogo.

Valadão Júnior, V. M., \& Mendonça, J. M. B. (2015). Assédio moral no trabalho: dilacerando oportunidades. Cadernos EBAPE.BR, 13(1), 19-39. Doi: 10.1590/1679-39519022.

Venco, S. (2006). Centrais de atendimento: a fábrica do século XIX nos serviços do século XXI. Revista Brasileira de Saúde Ocupacional, 31(114), 7-18. Doi: 10.1590/s0303-76572006000200002.

Vieira, C. E. C., Barros, V. A., \& Lima, F. de P. A. (2007). Uma abordagem da Psicologia do Trabalho, na presença do trabalho. Psicologia em Revista, 13(1), 155-168.

Vieira, C. E. C., Lima, F. de P. A., \& Lima, M. E. A. (2012). E se o assédio não fosse moral?: perspectivas de análise de conflitos interpessoais em situações de trabalho. Revista Brasileira de Saúde Ocupacional, 37(126), 256-268. Doi: 10.1590/s0303-76572012000200007.

Xavier, A. C. H., Barcelos, C. R. V., Lopes, J. P., Chamarelli, P. G., Ribeiro, S. de S., Lacerda, L. da S., \& Palacios, M. (2008). Assédio moral no trabalho no setor de saúde no Rio de Janeiro: algumas características. Revista Brasileira de Saúde Ocupacional, 33(117), 1522. Doi: 10.1590/s030376572008000100003 . 
Recebido em: $1^{\circ} / 8 / 2017$

Aprovado em: 2/3/2019 